\title{
A novel digital calibration technique for pipelined ADCs
}

\author{
Tohid Moosazadeh ${ }^{\mathrm{a})}$ and Mohammad Yavari $^{\mathrm{b}}$ ) \\ Integrated Circuits Design Laboratory, Department of Electrical Engineering, \\ Amirkabir University of Technology, Tehran, Iran \\ a)tmoosazadeh@aut.ac.ir \\ b) myavari@aut.ac.ir
}

\begin{abstract}
This paper presents a digital background calibration technique to correct the capacitors mismatch, gain error and gain nonlinearities of $1.5 \mathrm{bit} / \mathrm{stage}$ pipelined ADCs. The calibration technique uses a modified structure for the ADC stages, the skip-fill method and LMS algorithm and does not require any accurate calibration signal and any added analog circuitry; just some digital circuits are needed to fill the skipped samples and realize the LMS algorithm. Circuit level simulation results in a $90-\mathrm{nm}$ CMOS technology are provided for a 12 bit $80-\mathrm{MS} / \mathrm{s}$ pipelined ADC to verify the effectiveness of the proposed calibration technique.
\end{abstract}

Keywords: pipelined ADCs, capacitor mismatch, gain error, amplifier nonlinearity, digital background calibration, skip-fill method

Classification: Integrated circuits

\section{References}

[1] C. Grace, P. J. Hurst, and S. H. Lewis, "A 12-bit 80-MSample/s pipelined ADC with bootstrapped digital calibration," IEEE J. Solid-State Circuits, vol. 40, no. 5, pp. 1038-1046, May 2005.

[2] B. Sahoo and B. Razavi, "A 12-bit 200-MHz CMOS ADC," IEEE J. Solid-State Circuits, vol. 44, no. 9, pp. 2366-2380, Sept. 2009.

[3] A. Verma and B. Razavi, "A 10-Bit 500-MS/s 55-mW CMOS ADC," IEEE J. Solid-State Circuits, vol. 44, no. 11, pp. 3039-3050, Nov. 2009.

[4] A. Panigada and I. Galton, "A $130 \mathrm{~mW} 100 \mathrm{MS} / \mathrm{s}$ pipelined ADC with 69 dB SNDR enabled by digital harmonic distortion correction," IEEE J. Solid-State Circuits, vol. 44, no. 12, pp. 3314-3328, Dec. 2009.

[5] U. K. Moon and B. S. Song, "Background digital calibration techniques for pipelined ADCs," IEEE Trans. Circuits Syst. II, vol. 44, pp. 102-109, Feb. 1997.

[6] S. S. Haykin, Adaptive Filter Theory, Prentice-Hall, Upper Saddle River, 1996.

[7] L. Sumanen, M. Waltari, V. Hakkarainen, and K. Halonen, "CMOS dynamic comparators for pipeline ADC," Proc. ISCAS, vol. 5, pp. 157-160, May 2002.

[8] M. Dessouky and A. Kaiser, "Very low-voltage digital-audio $\Sigma \Delta$ modulator with 88-dB dynamic range using local switch bootstrapping," IEEE J. Solid-State Circuits, vol. 36, pp. 349-355, March 2001. 


\section{Introduction}

The design of high-speed, high-resolution analog-to-digital converters (ADCs) is a key issue in high-performance digital communication systems. The pipelined ADCs can produce such speeds but the design of high precision analog elements becomes more complicated as the device dimensions and supply voltages are scaled down. The capacitors mismatch, gain error, and gain nonlinearity are the main precision limitation factors for high-resolution pipelined ADCs in deep-submicron low-voltage CMOS technologies [1, 2].

A common way to overcome these precision limitations is to apply a calibration method to the converter. Recently, a few digital calibration techniques that cancel the precision limitations of pipelined ADCs have been proposed $[1,2,3,4]$. In these methods, the errors are generally measured using a calibration signal where the precision of this signal in deep-submicron technologies is an important issue. An accurate DAC calibrated with an additional calibration cycle is used in [1] to produce accurate calibration signals where in [2] with driving the mean square of 128 local errors across the input range to zero, they are measured using a medium-precision calibration signal. A high-precision on-chip DAC is used for 10-bit resolution in [3] and in [4] an accurate calibration signal produced with the DAC noise cancellation technique is used. Here, an efficient method is proposed which uses the lowest extra analog elements without the need for an accurate calibration signal.

\section{ADC errors modeling}

A pipelined ADC is composed of several low-resolution stages. Commonly, the 1.5-bit/stage architecture is used for high-speed pipelined ADCs [3]. Figure 1 (a) shows a single-ended switched-capacitor circuit implementation of the 1.5-bit stage with a capacitor non-flip-around multiplying digital-toanalog converter (MDAC). During $\phi_{1}$, the stage input signal is sampled and then during $\phi_{2}$, the difference of input signal and analog equivalent of the sub-ADC output $\left(V_{d a c}\right)$ is amplified. This configuration is chosen to simplify the modeling of the ADC stages and the calibration process like [1,2].

As discussed in [2], the inverse function of the ADC stages with the abovementioned structure in digital domain can be approximated as a third-order polynomial function given by:

$$
D_{\text {in }}=D+\beta_{1} D_{\text {out }}+\beta_{3} D_{\text {out }}^{3}
$$

where $D_{\text {in }}$ and $D_{\text {out }}$ are the digital equivalent of stage input and output, respectively, and $D$ is the stage sub-ADC output. The stage capacitors mismatch, gain errors, and gain nonlinearities of the amplifier are considered in the model with the $\beta_{1}$ and $\beta_{3}$ coefficients [2]. Having known $\beta_{1}$ and $\beta_{3}$ in (1) for all stages, the inverse function of $\mathrm{ADC}$ in digital domain is calculated and thus its input signal can be digitized without any dependency to the analog elements preciseness. 


\section{Proposed calibration technique}

The goal of the proposed calibration technique is the measurement of coefficients in (1) by using constant calibration signals without any dependency to their accuracy. The ADC uses 14 1.5-bit stages and a 2-bit flash ADC as last stage to achieve 12-bit resolution. In the stages 1 and 2, both linear and nonlinear terms in (1) are considered where in stages 3 to 14 only the linear term is considered $\left(\beta_{3}=0\right)$. Each calibration cycle begins with the $14^{\text {th }}$ stage and is done stage by stage up to the first stage. The background calibration was performed by skip-fill method proposed in [5]. As shown in Fig. 1 (b), for the $i^{\text {th }}$ stage background calibration, the ADC input sample is skipped at $n_{0} T_{S}$ and then the calibration signal, $V_{c a l}$, is inserted to this stage in the produced time slot at $\left[n_{0}+0.5(i-1)\right] T_{S}$ where $T_{S}$ and $n_{0}$ are the sampling period and time index respectively. So, in the proposed technique, the calibration is performed at the created time slots without interrupting the ADC normal operation.

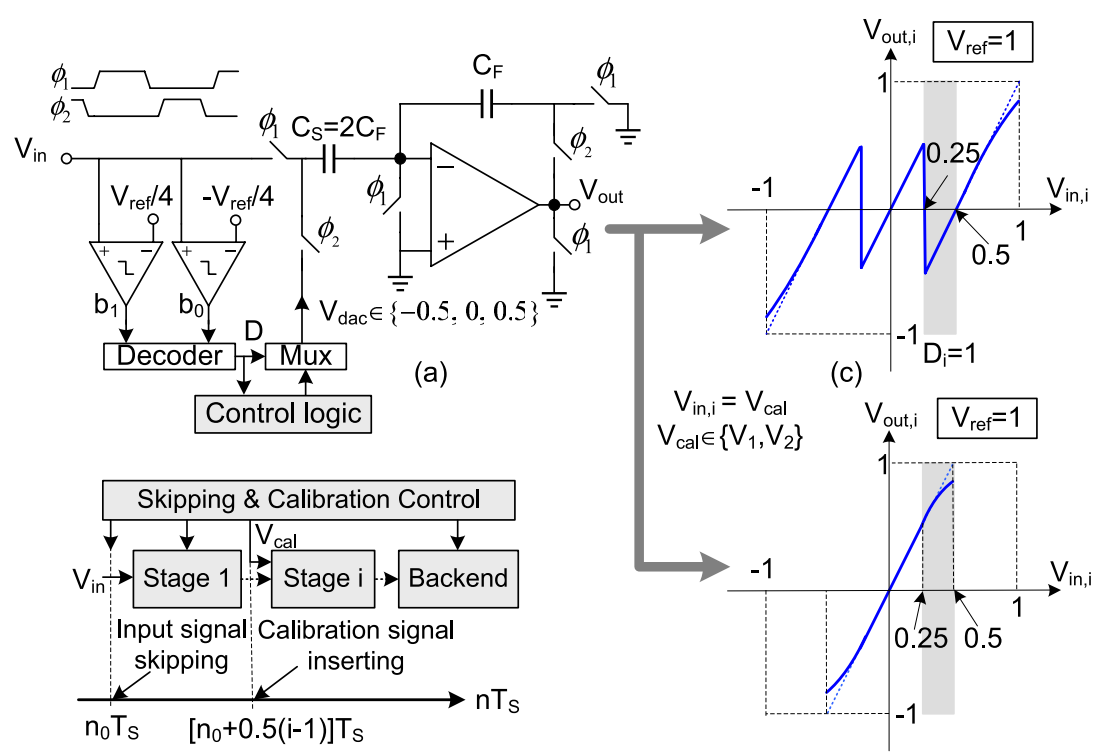

(b)

(d)

Fig. 1. (a) A 1.5 bit stage with capacitor non-flip-around MDAC, (b) background implementation of calibration, (c) the $i^{\text {th }}$ stage transfer function in 1.5bit mode, and (d) the $i^{\text {th }}$ stage transfer function in multiply-by-two mode.

To calibrate $\beta_{1}$ for the stages 3 to 14 , the same concept as [1] is used to measure the stage transfer function gaps in the comparators threshold voltage, $\pm 0.25 V_{\text {ref }}$. If the stage output is accurately digitized by the backend stages, then for $i=3$ to 14 , we have $D_{i n, i}=D_{i}+\beta_{1 i} D_{\text {out }, i}$, where $D_{i n, i}$ and $D_{\text {out }, i}$ are the digital equivalent of the $i^{\text {th }}$ stage input and output, respectively, $D_{i}$ is its sub-ADC output and $\beta_{1 i}$ is the linear term coefficient in $i^{\text {th }}$ stage inverse function.

To implement the proposed technique in these stages assuming zero offset 
comparators, each stage must be configured as two different modes in the calibration. In the first mode, with skipping a sample in the ADC input, the $i^{\text {th }}$ stage is configured as 1.5-bit mode at the appropriate time as shown in Fig. 1 (c) and a constant calibration signal, $V_{c a l}=V_{1}$, is applied to the $i^{\text {th }}$ stage input. $V_{1}$ is produced by a resistor ladder which is chosen with low precision in the range $0.25 V_{\text {ref }}<V_{1}<0.5 V_{\text {ref }}$ (so $V_{d a c}=0.5 V_{\text {ref }}$ ) and near the stage comparator threshold voltage same as [1] concept. Then, $D_{i}$ and the digitized output with backend stages in this mode, $D_{\text {out } 1, i}$, are stored in a memory. In the second mode, as shown in Fig. 1 (d), with skipping another sample in the ADC input, the $i^{\text {th }}$ stage is configured as multiply-by-two mode $\left(V_{d a c}=0\right)$ and the digitized stage output for $V_{1}$ with backend stages, $D_{\text {out } 2, i}$, is also stored in a memory. Indeed, in the multiply-by-two mode, the $i^{\text {th }}$ stage is configured as a sample and hold and $D_{i}$ is not used. With these two stored data and from (2), the digital equivalent of $V_{1}$ for the $i^{t h}$ stage in these two modes, $D_{i n 1, i}$ and $D_{i n 2, i},\left(D_{i n 1, i}=D_{i n 2, i}\right)$ are as follows:

$$
\left\{\begin{array}{l}
D_{\text {in } 1, i}=D_{i}+\beta_{1 i} D_{\text {out } 1, i} \\
D_{\text {in } 2, i}=\beta_{1 i} D_{\text {out } 2, i}
\end{array}\right\} \Rightarrow \beta_{1 i}\left(D_{\text {out } 2, i}-D_{\text {out } 1, i}\right)=D_{i} .
$$

Therefore $\beta_{1 i}$ can be extracted without any dependency on $V_{1}$. Moreover, it must be considered that when the $i^{\text {th }}$ stage is in the calibration process, the backend stages have been calibrated and produce an acceptable accurate digital equivalent for this stage output.

By extending the applied technique, both $\beta_{1}$ and $\beta_{3}$ for stages 1 and 2 , can also be extracted. In addition to $V_{1}$, another calibration signal, $V_{\text {cal }}=$ $V_{2}$, is applied to these stages. $V_{2}$ is also produced by the mentioned resistor ladder in the range $0.25 V_{\text {ref }}<V_{2}<0.5 V_{\text {ref }}$ and should be greater than $V_{1}$ as well as possible to measure the variation in amplifier gain caused by large amplitude input. However, with full-scale calibration signal ( $0.5 V_{\text {ref }}$ for multiply-by-two mode), the nonzero amplifier offset would cause over ranging. To maximize $V_{2}$ amplitude while avoiding such over ranging, the calibration signal is chosen near the stage DAC reference voltage, $0.5 V_{\text {ref }}$. In this condition, to extract $\beta_{1}$ and $\beta_{3}$, according to the explained sample skipping algorithm, $V_{1}$ and $V_{2}$ are applied in the two different mentioned modes to the stage input and the results are stored in the memory. Hence, from (1) we have:

$$
\begin{aligned}
& \left.V_{\text {in }, i}\right|_{i=1,2} \in\left\{V_{1}, V_{2}\right\}:\left\{\begin{array}{l}
D_{\text {in } 1, i}=D_{i}+\beta_{1 i} D_{\text {out } 1, i}+\beta_{3 i} D_{\text {out } 1, i}^{3} \\
D_{\text {in } 2, i}=\beta_{1 i} D_{\text {out } 2, i}+\beta_{3 i} D_{\text {out } 2, i}^{3}
\end{array}\right\} \\
& \Rightarrow \beta_{1 i}\left(D_{\text {out } 2, i}-D_{\text {out } 1, i}\right)+\beta_{3 i}\left(D_{\text {out } 2, i}^{3}-D_{\text {out } 1, i}^{3}\right)=D_{i} .
\end{aligned}
$$

The least mean square (LMS) algorithm [6] is used to simplify the required digital hardware for solving (2) and (3) as follows:

$$
\begin{aligned}
& \beta_{1 i}(n+1)=\beta_{1 i}(n)+\mu_{1} e(n)\left(D_{\text {out } 2, i}-D_{\text {out } 1, i}\right) \\
& \beta_{3 i}(n+1)=\beta_{3 i}(n)+\mu_{3} e(n)\left(D_{\text {out } 2, i}^{3}-D_{\text {out } 1, i}^{3}\right)
\end{aligned}
$$

where $\mu_{1}$ and $\mu_{3}$ are the LMS update step size in the coefficients extraction and $e(n)$ is given by:

$$
e(n)=D_{i}-\beta_{1 i}(n)\left(D_{\text {out } 2, i}-D_{\text {out } 1, i}\right)-\beta_{3 i}(n)\left(D_{\text {out } 2, i}^{3}-D_{\text {out } 1, i}^{3}\right)
$$


where for stages 3 to 14 is used with $\beta_{3 i}=0$. The accuracy of $V_{2}$ would not limit our calibration performance because it is enough that $V_{2}$ be less than $0.5 V_{\text {ref }}$ to avoid over ranging. Moreover, about $V_{1}$ accuracy it must be explained that the comparator offset can limit the range of $V_{1}$. To avoid this problem, the multiplexer used in the sub-DAC realization controls the stage mode in the $V_{1}$ insertion. For zero offset comparators of $i^{\text {th }}$ stage, in the 1.5 bit stage mode, $V_{1}$ produces a sub-ADC output of $D_{i}=1$ and $V_{d a c}=0.5 V_{\text {ref }}$, otherwise the sub-ADC has offset error $\left(D_{i}=0, V_{d a c}=0\right)$ and to calibrate the stage accurately, in the second insertion of $V_{1}$, it is configured with $V_{d a c}=0.5 V_{\text {ref }}$ using mode control logic, although $D_{i}=0$.

\section{ADC circuit implementation details}

To evaluate the performance of the proposed calibration technique, a 12-bit $80 \mathrm{MS} / \mathrm{s}$ pipelined ADC has been designed in the circuit level using standard 90-nm CMOS technology with $1-\mathrm{V}$ supply voltage. In the designed ADC, the input sample and hold circuit is eliminated with time constant matching of existing paths in the first stage. With thermal noise considerations, the stages 1 to 4 have $4 \mathrm{pF}, 1.8 \mathrm{pF}, 0.9 \mathrm{pF}$ and $0.4 \mathrm{pF}$ sampling capacitors, respectively, and the rest of the stages use $0.2 \mathrm{pF}$ sampling capacitor where maximum $0.1 \%$ capacitor mismatch is considered in each stage. A two-stage Miller compensated amplifier with two cascaded common-source amplifiers is used as the ADC amplifiers where its open loop DC gain is only $38 \mathrm{~dB}$ with maximum closed-loop gain variation of $10 \mathrm{LSB}$ of 12 bit resolution. The charge-distribution structure with a dynamic latch is used as the ADC comparators [7]. In addition, the bootstrapping technique is used in the sampling switches needing high linearity [8]. For skipped samples recovering, an 80taps FIR filter is used to realize the nonlinear Lagrange interpolation.

\section{Simulation results}

The analog circuits of ADC are simulated with HSPICE while the calibration process is implemented with MATLAB. Figures $2(\mathrm{a})$ and $2(\mathrm{~b})$ plot the simulated DNL and INL before and after the calibration, respectively. The uncalibrated ADC has a peak INL of 45 LSB where after calibration, the peak INL falls below 0.25 LSB. Figure 2 (c) shows the simulated output spectrum for a $1 \mathrm{MHz}, 1.2 \mathrm{-V}$ peak-to-peak differential analog input sampled at $80 \mathrm{MHz}$ where the SNDR and SFDR improvements are over $35 \mathrm{~dB}$ and $39 \mathrm{~dB}$, respectively. For survey of ADC performance in the Nyquist band, the SNDR and SFDR are calculated for several input signal frequency. The simulation results are shown in Fig. 2 (d). The peak SNDR is equal to $73 \mathrm{~dB}$ after calibration for an input frequency of $10 \mathrm{MHz}$. Each stage calibration time is about $2^{12}$ ADC sampling periods, so the total calibration time is about $14 \times 2^{12}$ ADC sampling periods. The achieved performance for the pipelined ADC and the comparison with recently reported ADCs is shown in Table I using a figure of merit (FoM) defined by $F o M=\frac{P o w e r}{2^{E N O B} f_{S}} V_{D D}$ as [4], where Power, $f_{S}$ and $V_{D D}$ refer to the ADC power consumption, sampling 
frequency and supply voltage respectively and $E N O B$ shows its effective number of bits. The simulated ADC shows the lowest FoM verifying the usefulness of the proposed calibration technique in design of high-speed and high-resolution pipelined ADCs.

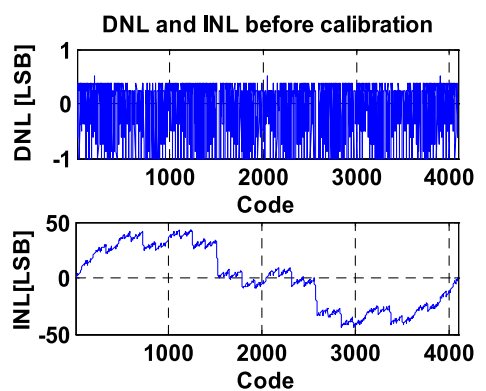

(a)

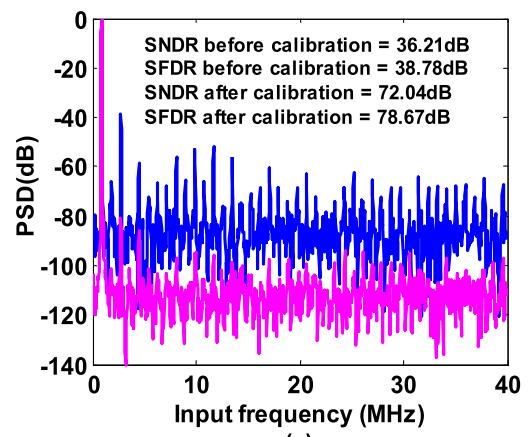

(c)

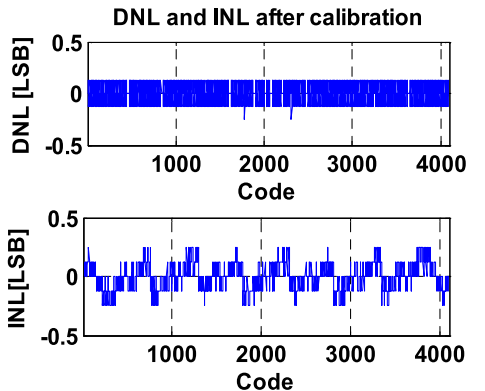

Code
(b)

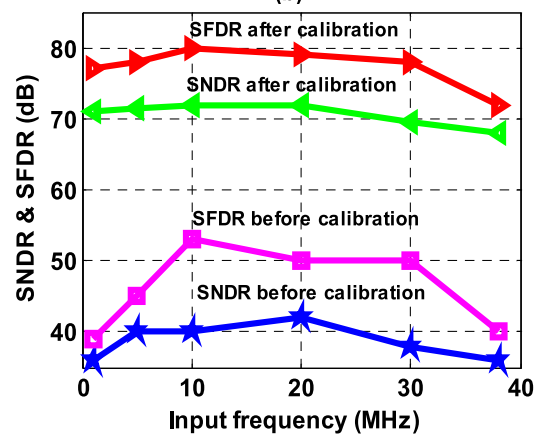

(d)

Fig. 2. (a) INL and DNL before calibration, (b) INL and DNL after calibration, (c) output spectrum before and after calibration for $0 \mathrm{dBFS}$ input signal, and (d) SNDR and SFDR vs. ADC input signal frequency.

Table I. Performance comparison of the simulated ADC.

\begin{tabular}{|c|c|c|c|c|c|c|c|c|c|}
\hline Ref. & Process & $\begin{array}{c}\mathbf{V}_{\text {DD }} \\
(\mathbf{V})\end{array}$ & $\begin{array}{c}\mathbf{f}_{\mathbf{S}} \\
(\mathbf{M S} / \mathbf{s})\end{array}$ & $\begin{array}{c}\text { INL } \\
(\mathbf{L S B})\end{array}$ & $\begin{array}{c}\text { DNL } \\
(\mathbf{L S B})\end{array}$ & $\begin{array}{c}\text { SFDR } \\
(\mathbf{d B})\end{array}$ & $\begin{array}{c}\text { SNDR } \\
(\mathbf{d B})\end{array}$ & $\begin{array}{c}\text { AnalogPower } \\
(\mathbf{m W})\end{array}$ & $\begin{array}{c}\text { FoM } \\
(\mathbf{p J} . \mathbf{V} / \mathbf{s t e p})\end{array}$ \\
\hline This Work & $90 \mathrm{~nm}$ & 1 & 80 & \pm 0.25 & -0.25 & 78 & 72 & 57.8 & 0.22 \\
\hline$[1]$ & $0.25 \mu \mathrm{m}$ & 2.5 & 80 & +0.24 & +0.09 & 84.5 & 72.6 & 340 & 3 \\
\hline$[2]$ & $90 \mathrm{~nm}$ & 1.2 & 200 & +1.3 & +0.59 & - & 64 & 348 & 1.6 \\
\hline$[3]$ & $90 \mathrm{~nm}$ & 1.2 & 500 & 1 & 0.4 & - & 52 & 55 & 0.4 \\
\hline$[4]$ & $90 \mathrm{~nm}$ & 1.2 & 100 & 3.6 & 0.54 & 85 & 69.5 & 93 & 0.44 \\
\hline
\end{tabular}

\section{Conclusion}

A digital background calibration technique was proposed to correct the capacitors mismatch, gain error and gain nonlinearities of the amplifiers in the $1.5 \mathrm{bit} / \mathrm{stage}$ pipelined ADCs. The proposed algorithm does not require any accurate calibration signal and is only realized by digital circuits. The circuit level simulation results show the effectiveness of the proposed calibration technique.

(c) IEICE 2010

DOI: 10.1587/elex.7.1741 Received October 10, 2010 Accepted November 04, 2010 Published December 10, 2010 